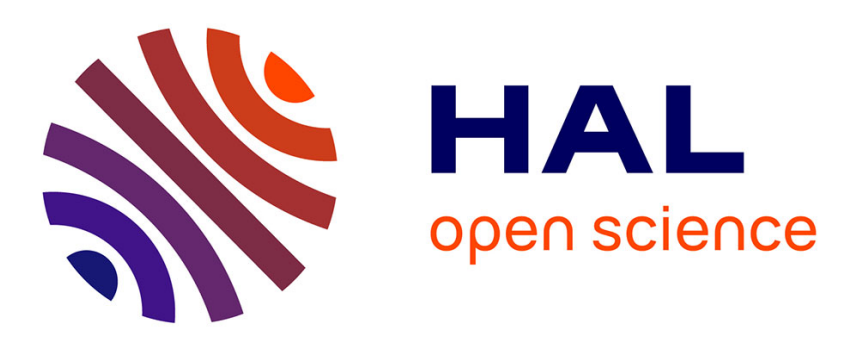

\title{
Méthode d'intégration électronique pour la mesure d'aimantation de supraconducteurs de faible volume et de faible champ critique
}

\author{
A. Nemoz, J.C. Solecki, J.P. Faure, J.R.G. Keyston
}

\section{To cite this version:}

A. Nemoz, J.C. Solecki, J.P. Faure, J.R.G. Keyston. Méthode d'intégration électronique pour la mesure d'aimantation de supraconducteurs de faible volume et de faible champ critique. Revue de Physique Appliquée, 1969, 4 (1), pp.42-44. 10.1051/rphysap:019690040104200 jpa-00242888

\section{HAL Id: jpa-00242888 https://hal.science/jpa-00242888}

Submitted on 1 Jan 1969

HAL is a multi-disciplinary open access archive for the deposit and dissemination of scientific research documents, whether they are published or not. The documents may come from teaching and research institutions in France or abroad, or from public or private research centers.
L'archive ouverte pluridisciplinaire HAL, est destinée au dépôt et à la diffusion de documents scientifiques de niveau recherche, publiés ou non, émanant des établissements d'enseignement et de recherche français ou étrangers, des laboratoires publics ou privés. 


\title{
MÉTHODE D'INTÉGRATION ÉLEGTRONIQUE POUR LA MESURE D'AIMANTATION DE SUPRAGONDUGTEURS DE FAIBLE VOLUME ET DE FAIBLE GHAMP GRITIQUE
}

\author{
Par A. NEMOZ, J. G. SOLEGKI, J. P. FAURE et J. R. G. KEYSTON (1), \\ Gentre de Recherches sur les Très Basses Températures, G.N.R.S., Grenoble, France.
}

(Reçu le 14 octobre 1968.)

\begin{abstract}
Résumé. - On décrit la réalisation d'un montage de mesure d'aimantation par intégration avec amplification préalable, qui permet d'obtenir, pour des supraconducteurs de faible volume et de faible champ critique, le tracé direct de la courbe d'aimantation, et en particulier les sauts d'aimantation, sur un enregistreur XY rapide.
\end{abstract}

Abstract. - An electronic integration device with preamplification for magnetization measurements is described. Magnetization curves, even with flux jumps, can be traced for superconducting materials of small volume, at low critical fields, on a fast XY recorder.

I. Introduction. - La méthode classique de mesure d'aimantation dite " par extraction » [1] consiste à déplacer l'échantillon soumis à un champ magnétique constant à l'intérieur d'une bobine de mesure. La variation deflux engendrée, proportionnelle à l'aimantation, est mesurée à l'aide d'un galvanomètre balistique. La bobine est constituée de deux enroulements disposés bout à bout et bobinés en sens contraire. Le champ est uniforme sur toute la longueur de la bobine. Cette méthode, bien que précise, présente quelques inconvénients : elle est sensible à la dérive du champ appliqué lors de l'extraction, elle est lente, soumet l'échantillon à un choc et à une faible variation de température, et n'est pas très adaptée à l'étude des sauts d'aimantation à cause des variations discrètes du champ magnétique. Pour ces raisons, nous avons entrepris la construction d'un montage de mesure d'aimantation par intégration avec variation continue du champ. Mais à cause du faible volume des échantillons à étudier, quelques dizaines de $\mathrm{mm}^{3}$, et des faibles champs critiques, dans le domaine de température utilisé, quelques dizaines d'œrsteds, une amplification préalable est alors nécessaire.

II. Principe (fig. 1). - Le champ magnétique est créé par une bobine dont le courant varie linéairement en fonction du temps. Un signal proportionnel au courant est envoyé à l'entrée $\mathrm{X}$ d'un enregistreur $\mathrm{XY}$. L'échantillon est disposé à l'intérieur de l'un des deux enroulements de la bobine de mesure. La tension aux

(1) Adresse actuelle : National Research Council of Canada, Ottawa 7, Canada.

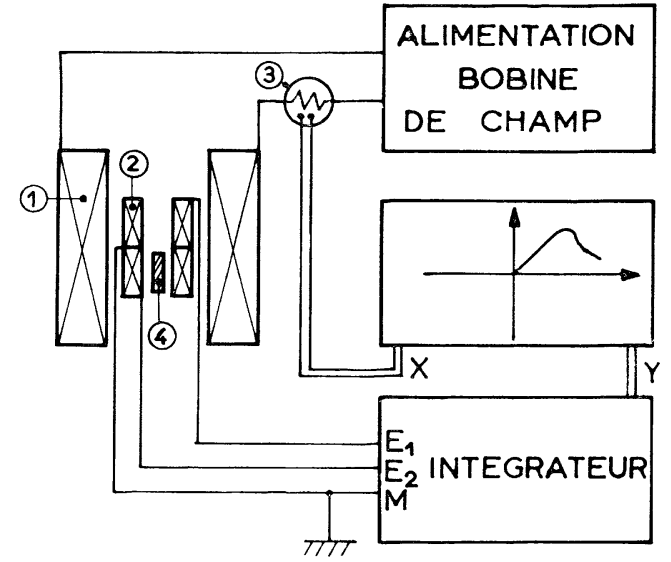

FIG. 1. - Schéma de principe : 1, Bobine de champ; 2, Bobine de mesure ; 3 , Résistance étalon ; 4, Échantillon.

bornes de la bobine, proportionnelle à la dérivée de l'aimantation, est d'abord amplifiée puis intégrée. Le signal obtenu est envoyé à l'entrée Y.

III. Réalisation. - Le montage cryogénique utilisé est un cryostat à double bain d'hélium [2] qui permet, par pompage direct sur le bain intérieur, où se trouve l'échantillon, d'obtenir une température de l'ordre de $0,75^{\circ} \mathrm{K}$, nécessaire pour nos mesures.

A. Champ magnétioue. - Le champ uniforme à $3 \times 10^{-3}$ près sur $8 \mathrm{~cm}$ est créé par une bobine $(H / I=187,8 \mathrm{E} / \mathrm{A})$, refroidie à l'azote liquide. Une 


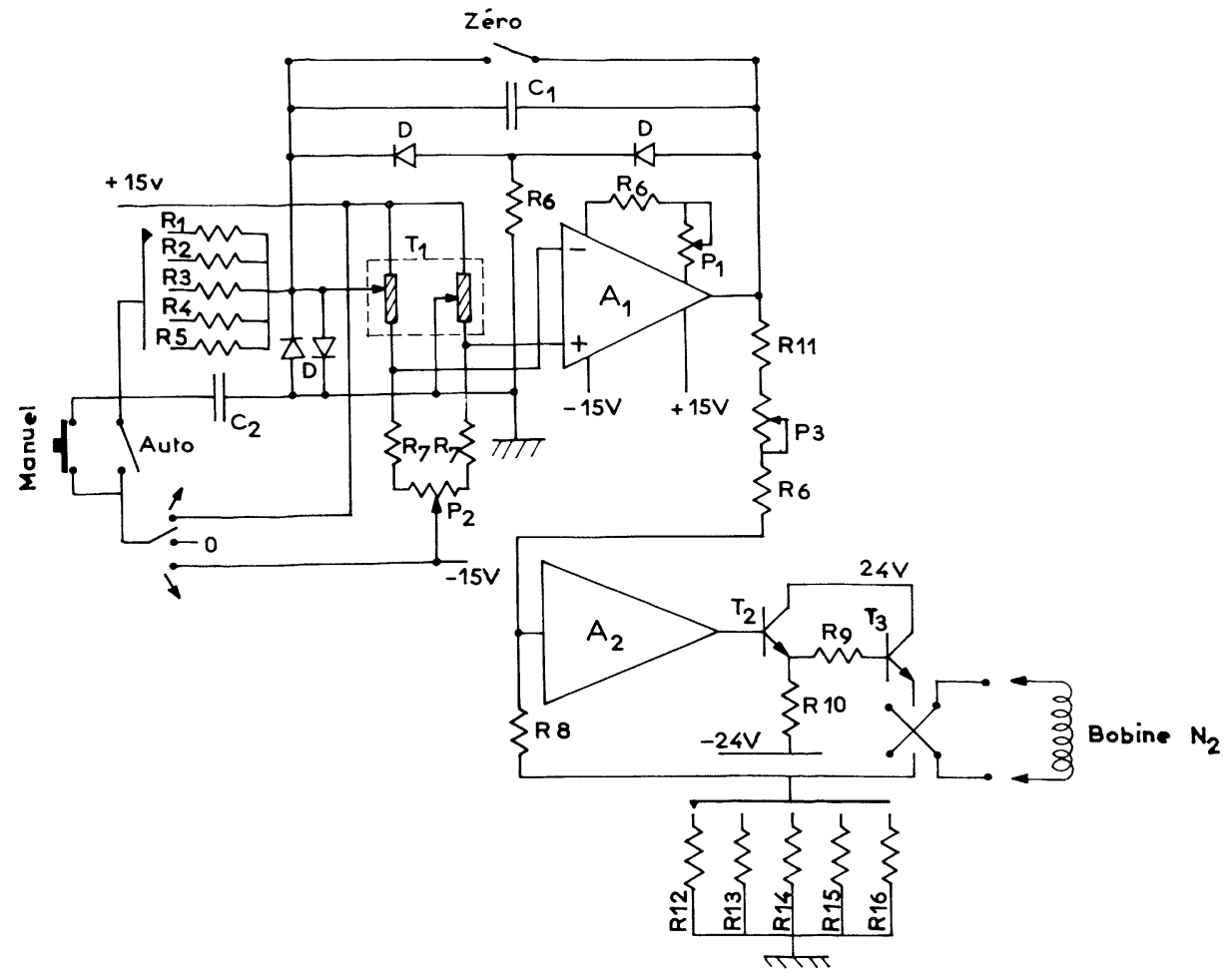

FIG. 2. - Schéma de l'alimentation de la bobine de champ. - Valeurs des composants: $\mathrm{A}_{1}$, Amplificateur Nexus SQ $10 ; \mathrm{A}_{2}$, Amplificateur continu; $\mathrm{T}_{1}$, T.E.C. $2 \mathrm{~N} 3958 ; \mathrm{T}_{2}, 2 \mathrm{~N} 1711 ; \mathrm{T}_{3}, 181 \mathrm{~T}_{2} ; \mathrm{D}, \mathrm{FD} 100$; $\mathrm{C}_{1}, 10 \mu \mathrm{F} ; \mathrm{C}_{2}, 10 \mathrm{kpF} ; \mathrm{P}_{1}, 22 \mathrm{k} \Omega ; \mathrm{P}_{2}, 4,7 \mathrm{k} \Omega ; \mathrm{P}_{3}, 10 \mathrm{k} \Omega ; \mathrm{R}_{1}, 3 \mathrm{M} \Omega ; \mathrm{R}_{2}, 6 \mathrm{M} \Omega ; \mathrm{R}_{3}, 12 \mathrm{M} \Omega ; \mathrm{R}_{4}, 24 \mathrm{M} \Omega ; \mathrm{R}_{5}, 66 \mathrm{M} \Omega ;$ $\mathrm{R}_{6}, 10 \mathrm{k} \Omega ; \mathrm{R}_{7}, 15 \mathrm{k} \Omega ; \mathrm{R}_{8}, 10 \mathrm{k} \Omega ; \mathrm{R}_{9}, 47 \Omega ; \mathrm{R}_{10}, 22 \mathrm{k} \Omega ; \mathrm{R}_{11}, 33 \mathrm{k} \Omega ; \mathrm{R}_{12}, 1,5 \Omega ; \mathrm{R}_{13}, 3 \Omega ; \mathrm{R}_{14}, 5 \Omega ; \mathrm{R}_{15}, 10 \Omega ;$ $\mathrm{R}_{16}, 15 \Omega$.

alimentation électronique ( $f g .2$ ) fournit un courant continûment variable depuis 0 jusqu'à une valeur maximale réglable de $200 \mathrm{~mA}$ à $2 \mathrm{~A}$. Le temps d'excursion peut varier de $30 \mathrm{~s}$ à $10 \mathrm{mn}$.

B. Intégration. - 1. Bobine de mesure. - La bobine est située dans le bain extérieur d'hélium, maintenu à $4,2 \mathrm{o}$. Chaque enroulement comporte 100000 spires de fil de cuivre de $7 / 100 \mathrm{~mm}$, réparties sur $6 \mathrm{~cm}$, de diamètre compris entre $26 \mathrm{~mm}$ et $52 \mathrm{~mm}$. Les deux enroulements sont identiques à une spire près. A cause des inhomogénéités de champ et de contractions non reproductibles lors du refroidissement, une compensation externe, décrite par ailleurs, est nécessaire. Les échantillons sont des alliages d'aluminium, Al-Ag ou $\mathrm{Al}-\mathrm{Mg}$ de température de transition de l'ordre de $1{ }^{\circ} \mathrm{K}$. Ils ont pour dimensions typiques : $20 \mathrm{~mm}$ de long, $8 \mathrm{~mm}$ de large et $1 \mathrm{~mm}$ d'épaisseur.

2. Amplification-intégration (fig. 3). - Les tensions délivrées, de l'ordre du $\mu \mathrm{V}$, sont amplifiées par un amplificateur à courant continu à gain réglable. Les deux entrées $E_{1}$ et $E_{2}$ correspondent chacune à un enroulement. Le potentiomètre $P_{1}(100 \Omega, 10$ tours $)$ permet de compenser l'enroulement qui délivre la tension la plus forte. Le deuxième étage est un intégrateur classique. Les deux amplificateurs $A_{1}$ et $A_{2}$ du type Fairchild A 006 ont une dérive de $0,1 \mu \mathrm{V} /$ semaine et de $0,5 \mu \mathrm{V} /{ }^{\circ} \mathrm{C}$, une largeur de bande de $1 \mathrm{MHz}$ et

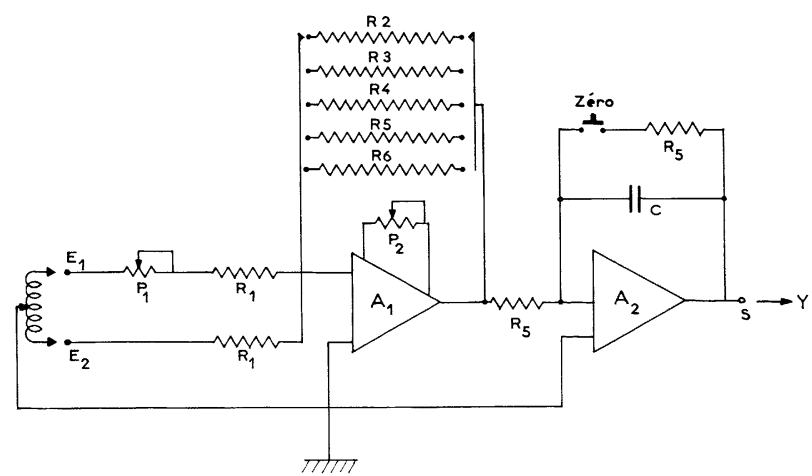

Fig. 3. - Schéma de l'intégrateur. - Valeurs des composants: $A_{1}, A_{2}$, Amplificateur Fairchild; $\mathrm{P}_{1}, 100 \Omega$ - 10 tours ; $\mathrm{P}_{2}, 100 \mathrm{k} \Omega-10$ tours ; $\mathrm{R}_{1}, 10 \mathrm{k} \Omega 0,5^{\circ}{ }_{\circ}^{\prime}$; $\mathrm{R}_{2}, 10 \mathrm{M} \Omega 0,5_{\circ}^{\circ} ; \mathrm{R}_{3}, 4,7 \mathrm{M} \Omega 0, \mathrm{5}^{\circ}{ }_{\circ}^{\prime} ; \mathrm{R}_{4}, 2,2 \mathrm{M} \Omega 0,5^{\circ}$; $\mathrm{R}_{5}, 1 \mathrm{M} \Omega 0,5{ }_{0}^{\circ} ; \mathrm{R}_{6}, 400 \mathrm{k} \Omega 0,50_{0}^{\circ} ; \mathrm{C}, 0,5 \mu \mathrm{F}$; $R_{\mathrm{f}}>10^{12} \Omega$. 
un gain de $5 \times 10^{7}$. Afin de réduire l'effet de dérive dû aux variations de température ambiante, l'ensemble amplification-intégration est plongé dans une enceinte maintenue à température constante par une circulation d'eau régulée en température. La dérive totale mesurée sur 10 minutes correspond ainsi à une tension ramenée à l'entrée de quelques nanovolts. Pour le cas où les tensions délivrées seraient importantes, le gain du premier étage peut être réduit afin de ne pas saturer l'intégrateur.

C. Remarques. - Il faut prendre des précautions particulières, lors du montage, pour éviter les forces thermo-électriques parasites, les boucles de masse. En l'absence d'échantillon, ou lorsque celui-ci est à l'état normal, on règle, à l'aide du potentiomètre $\mathrm{P}_{1}$, la compensation à $10^{-4}$ près. Avant chaque cycle, la dérive de l'intégrateur est éliminée au moyen du potentiomètre $\mathrm{P}_{2}$ qui ajuste le zéro de l'amplificateur $A_{1}$. Nous nous sommes assurés que le déphasage, entre le courant et le champ magnétique, dû aux parois métalliques du cryostat est négligeable aux vitesses de variation du champ utilisées. D'autre part, nous utilisons un enregistreur $\mathrm{XY}$ potentiométrique rapide.

IV. Performances. - La figure 4 montre une courbe d'aimantation isotherme typique. La sensibilité estimée est de 50 maxwells $\mathrm{t} / \mathrm{cm}$. La dérive est de l'ordre de $\pm 2,5$ maxwells $\mathrm{t} / \mathrm{cm} / \mathrm{mn}$. A l'aide d'une régulation [3] en température sur le bain intérieur, nous

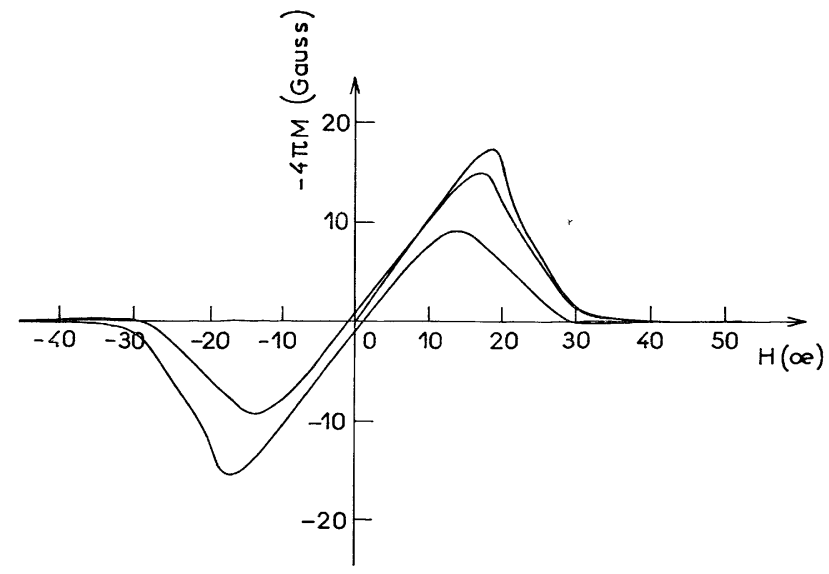

FIG. 4. - Courbe d'aimantation isotherme : Al, $4,12 \%$ At Ag $\left(T_{\mathrm{e}}=1,055 \pm 0,003 \circ \mathrm{K}\right), T=0,895 \circ \mathrm{K}$; $\frac{\mathrm{d} H}{\mathrm{~d} t}=1,9 \mathrm{CE} / \mathrm{s}^{-1}$. pouvons tracer un cycle tous les $10^{-2} \mathrm{oK}$. On jugera plus facilement des performances en comparant une courbe d'aimantation obtenue, d'une part, par la méthode d'extraction et mesure de la variation de flux à l'aide d'un galvanomètre (Kipp A 54) et, d'autre part, par la méthode ici décrite (fig. 5). L'accord est de l'ordre de 1 à $2 \%$. En outre, le cycle

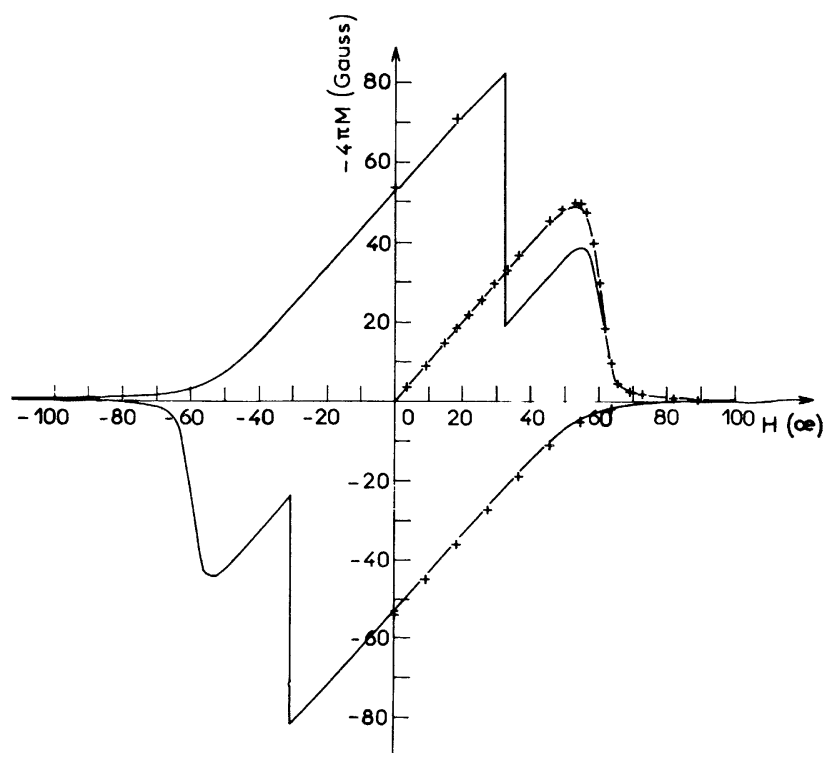

FIG. 5. - Courbe d'aimantation isotherme avec saut de flux : A1, $10 \%$ At $\mathrm{Mg}\left(T_{\mathrm{e}}=1,132 \circ \mathrm{K} \pm 0,003 \circ \mathrm{K}\right)$; $T=0,795 \circ \mathrm{K} ; \frac{\mathrm{d} H}{\mathrm{~d} t}=3,1 \quad \mathrm{E} / \mathrm{s}^{-1}$.

+ : Méthode d'extraction. : Méthode d'intégration.

comporte un saut d'aimantation qu'il était difficile de préciser par la première méthode. Ce montage semble donc adapté à l'enregistrement des variations rapides d'aimantation telles que les sauts d'aimantation. On peut aussi l'utiliser en méthode d'extraction, à la place du galvanomètre balistique, auquel cas la sensibilité est améliorée; on peut ainsi mesurer de très faibles aimantations.

V. Remerciements. - Les auteurs remercient MM. Tournier et Martin du Service d'Électronique Générale au Gentre d'Études Nucléaires de Grenoble pour leurs suggestions fort utiles et leur aide efficace. Ce travail a été réalisé avec l'aide de la Délégation Générale à la Rccherche Scientifique et Technique (D.G.R.S.T.).

\section{BIBLIOGRAPHIE}

[1] Shomnberg (D.), The Phys. Soc. (Cambridge), London, Conf. Report 2.85, 1967.

[2] Bonnin (B.), Thèse Faculté des Sciences, Université de Grenoble, 1965.

[3] KEYSTON (J.) et FAURE (J. P.), à paraître. 